\title{
Significado Trigonométrico en el Profesor
}

\author{
Teachers' Trigonometric Meaning
}

\author{
Gisela Montiel Espinosa* \\ Gonzalo Jácome Cortés ${ }^{* *}$
}

\begin{abstract}
Resumen
En este artículo presentamos el análisis de una experiencia con profesores del nivel medio superior en México, quienes trabajaron una situación-problema relacionada con el cálculo de distancias inaccesibles, en el contexto de un programa de actualización docente. Después de identificar en los profesores un significado lineal en la relación entre el ángulo y la distancia, nos planteamos como objetivo entender por qué se presenta este hecho. Bajo el encuadre de un marco conceptual que articula elementos cognitivos, didácticos y de construcción social de conocimiento trigonométrico, se analizaron los reportes entregados por cada profesor, posterior a la resolución de la situación-problema, así como algunos libros de texto. Identificamos que este significado lineal emerge de las actividades normadas por el discurso trigonométrico escolar, y que está inmerso en un fenómeno al que hemos llamado aritmetización de la Trigonometría.
\end{abstract}

Palabras-clave: Razón Trigonométrica. Discurso Matemático Escolar. Significado Trigonométrico.

\begin{abstract}
In this paper, we present the analysis of an experience with high school teachers in Mexico, who worked in a problem-situation related to inaccessible distance calculation in the context of a teacher's training program. After we identified a linear meaning in the relationship between the angle and distance, we set as our goal to understand why this fact is presented. Under the framework of a conceptual framework that articulates cognitive, educational, and social construction of knowledge trigonometric elements, we analyzed the reports submitted by each teacher after the resolution of the problem-situation and some textbooks. We identified that this linear meaning emerges from the activities regulated by the school trigonometric discourse, and is immersed in a phenomenon we call arithmetization of trigonometry.
\end{abstract}

Keywords: Trigonometric Ratio. Mathematic Scholar Discourse. Trigonometric Meaning.

\section{Introducción}

\footnotetext{
* Doctora en Matemática Educativa por el Instituto Politécnico Nacional. Profesora-Investigadora del Posgrado en Línea en Matemática Educativa del Centro de Investigación en Ciencia Aplicada y Tecnología Avanzada, Unidad Legaria del Instituto Politécnico Nacional, México DF. Dirección Postal: Calzada Legaria 694, Col. Irrigación CP 11500, Delegación Miguel Hidalgo. México, DF. E-mail: gmontiel@ipn.mx

** Maestro en Ciencias en Matemática Educativa por el Instituto Politécnico Nacional. Profesor del Telebachillerato de Veracruz. Dirección Postal: Mártires 28 de Agosto 212, Col. Obrero Campesina CP 91020. Xalapa, Veracruz. E-mail: gonzalojac321@ hotmail.com
} 
En las últimas décadas, la investigación sobre la enseñanza-aprendizaje de la Trigonometría ha encontrado gran variedad de concepciones y dificultades asociadas a los conceptos involucrados en esta área de la matemática escolar (GRABOVSKIJ; KOTEL'NIKOV, 1971; DE KEE; MURA; DIONNE, 1996; MALDONADO, 2005). Algunos resultados coinciden, probablemente porque el aprendizaje de las nociones trigonométricas es analizado como un continuo que inicia en la razón trigonométrica y culmina en la función trigonométrica. De esto, deviene natural que una parte significativa del debate sea sobre cuál es el método más adecuado para introducir a la Trigonometría, si el triángulo rectángulo o el círculo unitario (KENDAL; STACEY, 1998; WEBER, 2005, 2008).

En la investigación de Jácome (2011) profesores mexicanos del nivel medio superior, en un contexto de actualización docente, llevaron a cabo una experiencia didáctica cuya intención fue trabajar relaciones de proporcionalidad en la construcción de modelos geométricos para resolver una situación-problema tradicional de cálculo de distancias inaccesibles. El diseño de la situación-problema pone énfasis en la toma de medidas angulares y en la construcción de modelos geométricos a escala, a través de la experiencia y la manipulación. Es decir, no se les proporcionó una ilustración con medidas hipotéticas, sino que se les pidió localizar un objetivo (alto) en su entorno para calcular su altura.

En los reportes entregados por los profesores se pueden observar distintos fenómenos. Por ejemplo, quienes utilizan la razón trigonométrica tangente (RTT) como herramienta para resolver el problema hablan de función trigonométrica, función tangente, procedimiento trigonométrico, relación tangente, razones trigonométricas o, simplemente, fórmula tangente. En principio consideramos que debido a que en el nivel medio superior se trabaja con mucho más énfasis en las funciones trigonométricas esta ambigüedad entre razón (también vista como fórmula o procedimiento) y función es bastante frecuente.

Sin embargo, entre estos profesores se presentó un hecho (ver figura 1) que no se había atendido en la investigación sobre estos tópicos, y que resulta de nuestro interés porque pone en evidencia la necesidad de un rediseño del discurso matemático escolar que se sustente en la problematización de lo que estamos enseñando y no sólo de cómo lo estamos enseñando.

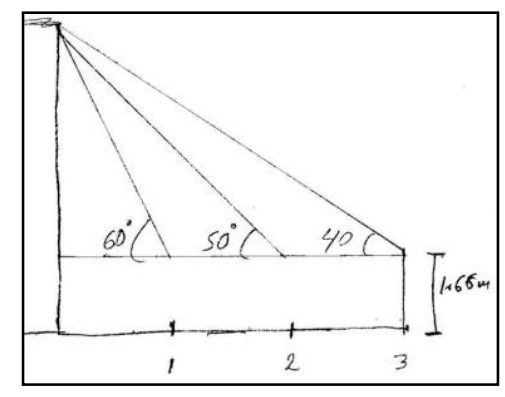


Figura 1- Ejemplo de significado lineal en la relación ángulo-distancia (cateto)

Los pasos que sigue el profesor para calcular la altura de un objetivo alto, tomando medidas a 1, 2 y 3 metros de distancia, son básicamente los de la tradición escolar. Sin embargo, la representación gráfica nos muestra que la costumbre didáctica de enfatizar la relación proporcional entre los lados del triángulo rectángulo pudiera sugerir, también, una relación lineal entre el ángulo y el cateto adyacente, cuando éste, para el caso de la experiencia, incrementa su medida en forma constante.

El objetivo de nuestro estudio es entender por qué se presenta este significado en la relación ángulo-distancia e identificar su origen. Partimos preguntándonos entonces ¿qué condiciona la actividad y la producción del profesor de matemáticas en una situaciónproblema de cálculo de distancias inaccesibles?

\section{Fundamento teórico}

Proponemos la articulación de tres planteamientos teóricos que nos permita estudiar la presencia del significado lineal que manifestaron los profesores en la resolución de una situación-problema de cálculo de distancias inaccesibles. Este significado lo asumiremos como asociado a la actividad humana, que se identifica a partir de la intención (implícita) conectada a la acción (KILPATRICK et al., 2005). De ahí que denominemos significado lineal a lo que subyace a la acción del profesor de plasmar una relación de (de)crecimiento constante en la relación ángulo-cateto adyacente en un triángulo rectángulo.

Las herramientas teóricas seleccionadas nos proporcionan elementos sobre el entendimiento del profesor, el contexto educativo en el que se desenvuelven y la naturaleza propia del saber matemático en juego.

\subsection{Racionalidad práctica del profesor de Trigonometría}

Herbst y Chazan (2011) caracterizan la racionalidad práctica como un contenedor de disposiciones, entendidas éstas en el sentido de Pierre Bourdieu como categorías de percepción y apreciación, que son viables en la enseñanza de las matemáticas para garantizar (o refutar) cursos de acción en ella. Con ello, los autores se proponen caracterizar las bases donde el profesor justifica sus acciones. Estas disposiciones tienen incidencia en un colectivo 
y están presentes para cumplir tanto con las normas de una situación instruccional específica, como con las obligaciones profesionales de la enseñanza de las matemáticas.

Muy en particular, en una situación instruccional de cálculo de valores de funciones trigonométricas Mesa y Herbst (2011) identifican que la racionalidad práctica del profesor justifica, al menos, tres normas:

(1) ninguno, profesor o estudiante, se responsabiliza por justificar los pasos de solución en un ejemplo o por explicar por qué una respuesta tiene sentido;

(2) los estudiantes no tienen control sobre los pasos a seguir cuando el instructor resuelve ejemplos en el pizarrón; y

(3) el instructor siempre proporciona suficiente información para resolver los problemas dados como ejemplo.

A partir del reconocimiento de estas normas, los autores identifican en la curricula del community college una percepción de la Trigonometría como proveedor de habilidades y conocimiento técnico para asignaturas más avanzadas, lo cual podría explicar por qué el profesor no invierte tiempo del curso en actividades distintas. Los autores consideran que esto influye para que las actividades didácticas tiendan a la repetición y a la modelación de procedimientos conocidos para que los estudiantes ganen familiaridad con el material, lo que eventualmente demostrará que han adquirido maestría con él.

\subsection{Construcción social de conocimiento trigonométrico}

El binomio Historia-Educación, referido a las matemáticas, ha encontrado diversas manifestaciones exitosas. Buendía y Montiel (2011) lo han utilizado para reconocer en la historia elementos que contribuyan en la explicación de fenómenos de aula y en el planteamiento de epistemologías de prácticas para la construcción de conocimiento matemático. Las autoras ponen en evidencia cómo, desde el enfoque teórico de la Socioepistemología, se desarrollan estrategias de investigación a través de la historia para determinar aquellas circunstancias que dan cuenta de por qué, hoy, tratamos con la matemática escolar como lo hacemos. En este enfoque social, la epistemología de prácticas propuesta por Montiel (2011) nace del estudio de las circunstancias históricas en las que se construye el conocimiento trigonométrico, y, a partir de ella, se distinguen tres momentos de construcción normados por la anticipación, la predicción y la formalización; y centra su atención en la emergencia de la relación, la funcionalidad y la formalidad trigonométricas, y sus respectivos 
desarrollos del pensamiento geométrico-proporcional, analítico-funcional y abstracto-formal; en contraste con la visión tradicional sobre el aprendizaje de la razón, la función y la serie.

Para efectos del presente artículo, profundizaremos, en el primer momento, aquel en donde emergen las razones trigonométricas como producto de la actividad humana.

La historia de la Trigonometría se construye sobre las aportaciones prácticas que le dieron origen en el marco de la astronomía, la geografía y a través de la construcción de grandes pirámides. Van Brummelen (2009) localiza los inicios del campo en los orígenes del sistema geocéntrico de Ptolomeo $(100$ - 170) e identifica un punto de ruptura significativo con el modelo heliocéntrico de Copérnico (1473-1543). Un estudio desde la Socioepistemología permite reconocer, en los usos prácticos, significados asociados a las nociones trigonométricas y elementos necesarios para construir las herramientas matemáticas que las representan.

Es en este periodo Montiel (2011) identifica a la anticipación como la práctica social que regula las actividades asociadas a la matematización de la astronomía, ya sea para la predicción o la explicación de fenómenos celestes, era necesario que éste sucediera para estar en condiciones de comprobar el dato y, a la vez, el modelo; la matematización, numérica o geométrica, orientaba las decisiones prácticas de la agricultura, el comercio o la navegación, del mismo modo que guiaba a las explicaciones teóricas de la astronomía o la geografía. Esto es, se tenía la necesidad de anticipación al fenómeno. Este acto anticipatorio preconfigura la emergencia de un conocimiento y en consecuencia, de un saber institucional.

La Trigonometría se constituye como un preliminar matemático de la teoría astronómica, que en virtud del dominio de la racionalidad helenística debía desarrollarse en el marco epistemológico que brindaba la geometría deductiva. La construcción de modelos a escala de una entidad real no manipulable (la inmensidad celeste) constituye una transición de lo macro a lo micro, donde la proporcionalidad entre ellos (realidad presente y realidad representada) condiciona la precisión del modelo. De manera natural, las razones se convierten en la abstracción inmediata de la proporción y los círculos, los arcos/ángulos y las cuerdas en los elementos constitutivos del modelo geométrico.

Con el objetivo de resaltar la logística implícita en el Almagesto de Ptolomeo, Pedersen (1974, p.35) describe los modelos geométricos que acompañan los desarrollos que, hoy, consideramos trigonométricos, como conceptos estáticos que se refieren a un instante particular en el tiempo. Para simular el movimiento del planeta, la configuración debía cambiar continuamente en el tiempo y la posición de cualquier punto definido en el tiempo se 
podía determinar por geometría ordinaria y calcular por los métodos trigonométricos descritos en el Almagesto. Pedersen reconoce que la Trigonometría se deriva de la Geometría y puede hacer una diferencia entre lo que le correspondería a cada una en el Almagesto, pero Ptolomeo lo desarrolla de forma integral y lo establece como presupuestos matemáticos para la astronomía teórica.

El momento en que las cuerdas del modelo ptolemaico se sustituyen por el seno de la astronomía de la India del siglo IV podría considerarse un paso significativo de la matemática-geométrica a la matemática-trigonométrica, es decir, un paso hacia la constitución de una rama del saber que, si bien se construye sobre la base de la lógica y del lenguaje euclidianos, nace, se desarrolla y consolida en estrecha relación con la observación de la naturaleza y el estudio de fenómenos no manipulables.

El conocimiento trigonométrico que se construye en este momento de anticipación, al estar vinculado a la matematización de la astronomía, está considerando una escala de tiempo finito, humana y cosmológica a la vez, cuyo periodo depende en definitiva del fenómeno específico en cuestión (día-noche, fases lunares, estaciones del año, eclipses, posición de un cuerpo celeste). Así, encontramos que la periodicidad y el valor de las cuerdas (valores acotados) estaban vinculados a la repetición de fenómenos astronómicos y a la posición de los cuerpos celestes, respectivamente, y una vez que eran encontrados periodo y posición, no había razón alguna para su estudio en tanto propiedad de la relación trigonométrica.

En estas circunstancias históricas se reconoce lo que es inherente al saber trigonométrico, desde la perspectiva de distintos grupos humanos, en el ejercicio de una práctica de referencia vinculada a explicar una realidad no manipulable.

\subsection{Discurso Matemático Escolar}

La noción de discurso Matemático Escolar (dME) que propone el enfoque teórico de la Socioepistemología permite considerar tanto las normas implícitas del contrato didáctico, como las invariantes aun en presencia de rupturas en dicho contrato, de ahí que nos sirve como articulador de los referentes previos.

Soto (2010) caracteriza al discurso matemático escolar (dME) como un sistema de razón que produce violencia simbólica, que al centrarse únicamente en el dominio de conceptos matemáticos ha generado la concepción de la Matemática como un conocimiento acabado, no suceptible de construirse, sino sólo de adquirirse. En consecuencia, estudiantes y 
profesores quedan excluidos de la construcción de conocimiento matemático, aun cuando no son exluidos del sistema escolar, de ahí que utilicemos esta noción para articular las consideraciones teóricas anteriores, porque señala a un fenómeno de permanencia y adaptación al sistema sin que necesariamente se construya conocimiento matemático. La racionalidad práctica del docente y una epistemología de lo trigonométrico basada en la actividad pueden dar luz de la existencia de este tipo de discurso, para el caso particular de la Trigonometría escolar.

El discurso matemático escolar subyace a lo inmediatamente visible. Tal como señalan Cantoral et al. (2006, p.86):

[...] la estructuración de dichos discursos no se reduce a la organización de los contenidos matemáticos, ni a su función declarativa en el aula (el discurso escolar), sino que se extiende un tanto más allá, al llegar al establecimiento de bases de comunicación para la formación de consensos y la construcción de significados compartidos.

El dME refleja una ideología sobre la forma de presentar y tratar (didácticamente) los objetos matemáticos en clase (CASTAÑEDA; ROSAS; MOLINA, 2010), y naturalmente se manifiesta y transmite en lo inmediatamente visible: Planes y Programas de Estudio, libros de texto, exposición de clase, o, como se ha identificado en estudios especializados, en las creencias y concepciones de profesores, estudiantes y comunidad académica en general.

En consecuencia es importante reconocer aquello que subyace a estas manifestaciones concretas del dME y permanece invariante aun con la innovación didáctica, debido a que en el fondo ésta no modifica lo que se está enseñando, sino sólo cómo se está enseñando. Para el caso de la presente investigación, una estrategia para reconocerlo es identificar el conjunto de significaciones asociadas al conocimiento trigonométrico porque estarían normando, de manera implícita o explícita, la actividad áulica y el discurso escolar mismo.

\section{Consideraciones metodológicas}

Con el objetivo de responder qué condiciona la actividad y la producción de nuestro colectivo de profesores, particularmente en la resolución una situación-problema de cálculo de distancias inaccesibles, analizamos dos fuentes de datos: los reportes de solución de cada profesor y el panorama curricular de la Trigonometría en el sistema educativo mexicano del nivel básico-secundaria.

Los profesores que participaron en la experiencia aquí reportada cuentan con perfil profesional afín a las matemáticas $\mathrm{y}$, entre muchas otras actividades, resolvieron una 
situación-problema intencionalmente diseñada para hacer emerger el uso de la proporcionalidad o de la razón trigonométrica tangente (RTT). Esta experiencia formó parte de los cursos de actualización y capacitación que reciben, periódicamente, los profesores del sistema de Telebachillerato al que pertenecen. Para resolver la situación-problema los profesores se organizaron en pequeños equipos de 3 ó 4 integrantes, y posteriormente entregaron un reporte individual; por lo que contamos con sus producciones en 42 reportes.

La segunda fuente de datos la constituyeron el Programa de Estudios y algunos libros de texto, avalados por la Secretaría de Educación Pública (SEP) de México, en donde se aborda el tema de las razones trigonométricas y que estaban vigentes en el periodo en que se llevó a cabo la experiencia docente que aquí se reporta. Los Planes y Programas de Estudio del Telebachillerato, que en ese momento iniciaba una reforma educativa, fueron analizados por Jácome (2011) y complementarían el panorama nacional que aquí presentamos con nuestro análisis didáctico. Para el análisis de esta fuente de datos realizamos una revisión de la organización didáctica que proponen y el tipo de actividades que pide al alumno, así como el contexto en el que las pide y el objetivo de hacerlas; es decir, buscamos responder a qué hace y por qué hace, para entender los significados que se van a construir a partir de llevar a cabo estas actividades didácticas.

Mostramos, primero, el análisis del dME con el propósito de retomarlo y articularlo en el análisis de las producciones del profesor, buscando evidenciar la coincidencia entre las actividades y herramientas que se desarrollan en él y las que refleja el profesor en sus reportes.

\subsection{La situación-problema}

La situación-problema pidió a los profesores una tarea clásica de cálculo de distancias inaccesibles, pero de manera vivencial, esto es, se les pidió localizar un objetivo (alto) en su entorno y calcular su altura. El diseño de la situación-problema pone énfasis, intencionalmente, en la toma de medidas angulares, desde diferentes posiciones (a $1 \mathrm{~m}, 2 \mathrm{~m}$ y $3 \mathrm{~m}$ del objetivo a medir), y en la construcción de un modelo geométrico para cada posición. Para realizar las mediciones se proporcionó a los profesores cinta métrica, tubo de cartón, regla, transportador, lápiz y cuaderno.

La intencionalidad de la instrucción Registra tus mediciones y realiza un modelo a escala de lo observado fue proporcionar a los profesores el contexto propicio para establecer 
relaciones de proporcionalidad entre la realidad y el modelo, que sirvieran para obtener la altura solicitada.

\subsection{Criterios de análisis para los reportes}

Con base en las regularidades mostradas en los reportes se construyeron cinco criterios de análisis de las producciones de los profesores, es decir, de lo que plasmaron en el reporte; considerando también las costumbres e implicaciones didácticas señaladas en nuestros fundamentos teóricos. En la Figura 2 mostramos los criterios, con algunos subcriterios para un análisis más detallado de las elecciones que hace el profesor para resolver la situación.

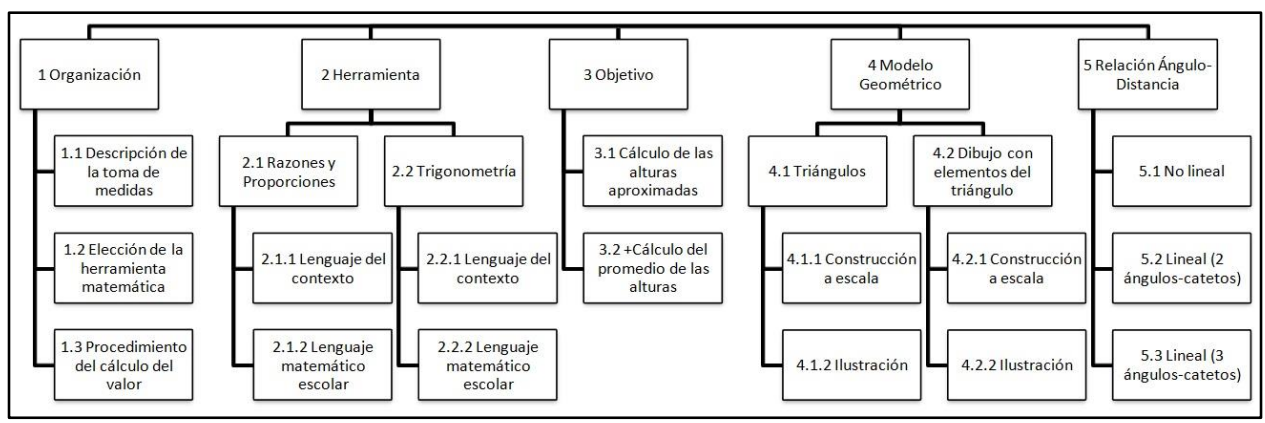

Figura 2- Criterios para el análisis de los reportes individuales

El primer criterio de análisis considera identificar patrones en la forma en cómo organizan el reporte de resolución, entendiendo que ello refleja el tipo de organización didáctica que los profesores consideran apropiada en la enseñanza-aprendizaje de la Proporcionalidad o la Trigonometría en el contexto del triángulo rectángulo. Debido a la interacción al interior de cada equipo, se espera que algunos reportes sean muy similares entre ellos, sin embargo, se contabilizan de manera individual porque se les reconoce como producto de un consenso, es decir, que al plasmarlo en el reporte personal están de acuerdo en que esa es la forma correcta de presentar lo trabajado como equipo.

El segundo criterio de análisis se basa en distinguir la herramienta matemática con la que los profesores resuelven la situación problema y hace diferencia entre elegir alguna de ellas utilizando de manera integrada el lenguaje típicamente escolar (tangente, cateto opuesto, cateto adyacente; en algunos casos sin unidades de medida) con el lenguaje del contexto de la situación-problema (altura, distancia, ángulo de inclinación; y sus correspondientes unidades de medida); o utilizándolos de manera desarticulada. Ejemplo de este último caso lo 
mostramos en la Figura 3, donde el profesor obtiene los valores en términos de $x$ (incógnita despejada de la fórmula de la razón trigonométrica tangente) y sus ilustraciones de referencia a la situación están en términos de $\mathrm{h}_{1} \mathrm{y} \mathrm{h}_{2}$; además de sumarle la altura del observador, a cada caso, sin hacer alguna alusión al origen de esta medida.

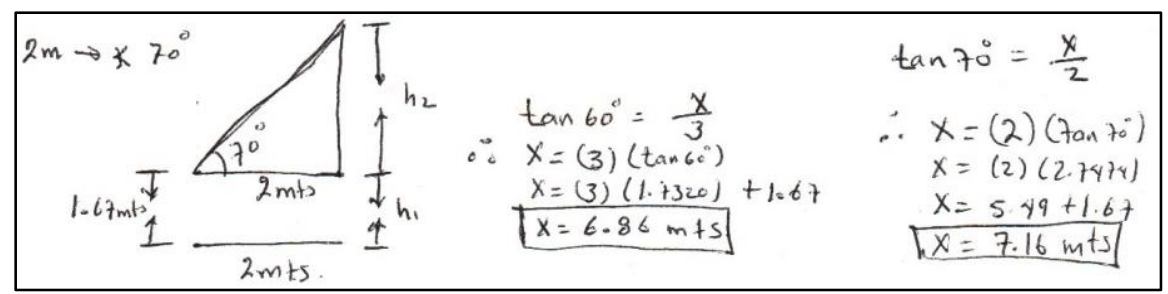

Figura 3- Uso desarticulado del lenguaje escolar y del lenguaje del contexto

Con el tercer criterio se hace una diferencia entre los reportes donde el profesor sólo calcula las tres alturas aproximadas y los reportes donde, además, promedia las alturas, intentando evidenciar hacia dónde se dirige el énfasis de la actividad matemática del profesor. El cuarto criterio distingue si el modelo geométrico en el reporte se compone sólo de triángulos o de un dibujo de la situación que se está modelando, en donde incluya elementos del triángulo, las distancias y los ángulos; distinguiendo, además, si los triángulos o los dibujos son representaciones a escala de la realidad. Finalmente, el quinto criterio distingue si el profesor plantea una relación no lineal o lineal entre el ángulo y las distancias a las que se sitúa cuando toma las medidas angulares, o no. Para considerar el subcriterio 5.2, donde se establezca la relación lineal entre dos mediciones, éstas deben ser continuas, es decir, a 1 y 2 metros o a 2 y 3 metros desde la posición donde el profesor toma la medida angular.

\subsection{Organización de los datos en los reportes}

Con base en estos criterios se trazaron trayectorias de cada reporte, indicando qué criterio y subcriterios cumple. Por ejemplo, en la siguiente tabla mostramos la trayectoria del reporte R2:

Tabla 1- Trayectoria del Reporte R2, indicando los subcriterios que cumple

\begin{tabular}{llll}
\hline \multicolumn{4}{c}{ R2 } \\
\hline $\mathbf{1}$ & 1.1 & 1.2 & 1.3 \\
\hline $\mathbf{2}$ & 2.2 & 2.2 .1 & \\
\hline $\mathbf{3}$ & 3.2 & & \\
\hline $\mathbf{4}$ & & 4.1 .2 & 4.2 .2 \\
\hline $\mathbf{5}$ & 5.3 & & \\
\hline
\end{tabular}

Esta trayectoria nos refleja que en el reporte R2 se hace una descripción de la toma de medidas que realizaron en equipo (subcriterio 1.1), se dice explícitamente qué herramienta va 
a utilizar para calcular la altura del edificio (subcriterio 1.2) y se desarrolla el procedimiento. Además de calcular las alturas para los tres casos solicitados, se calcula el promedio de ellas (subcriterio 3.2) para dar la altura del edificio. No se especifica cuál es el modelo geométrico, pero realiza tanto un dibujo del edificio, sobreponiéndole los elementos del triángulo (Figura 4, subcriterio 4.2.2), como un triángulo en la resolución de cada cálculo (Figura 5, subcriterio 4.1.2); aunque en ambos casos se trata de ilustraciones, no de reproducciones a escala.

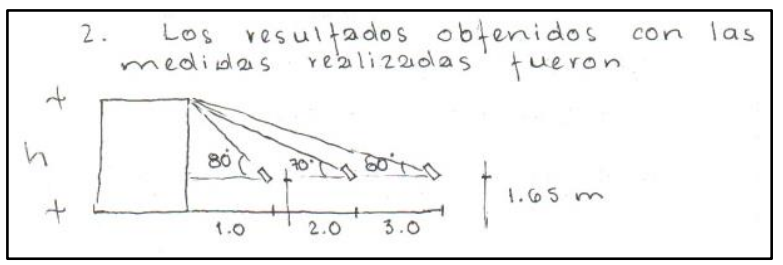

Figura 4- Dibujos representativo de la situaciónproblema (reporte R2)

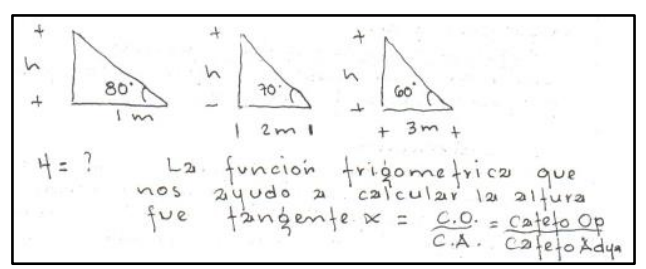

Figura 5- Triángulos ilustrativos para el cálculo de la altura faltante (reporte R2)

Finalmente, muestra una relación lineal entre los tres ángulos de elevación y las distancias desde donde realiza las mediciones angulares (subcriterio 5.3).

\subsubsection{Criterio 1: Organización didáctica}

Treinta y siete profesores (88\%) incluyen en el reporte una descripción de la actividad trabajada en equipo (subcriterio 1.1), detallan cómo se tomaron las medidas y algunos hacen una reflexión sobre por qué su inexactitud. Sobre la elección de la herramienta (subcriterio 1.2), treinta y seis profesores (85\%) señalan cuál les permitió hacer el cálculo, pero un número menor de ellos (treinta y tres profesores, 78\%) desarrollan el procedimiento matemático (subcriterio 1.3), paso a paso, del cálculo de la distancia.

Nueve reportes (21\%) cumplen sólo con el subcriterio 1.1, tres reportes (7\%) cumplen con los subcriterios 1.1 y 1.2 , cuatro reportes (9\%) cumplen con los subcriterios 1.2 y $1.3 ; \mathrm{y}$, finalmente, veintiocho reportes (66\%) cumplen con los tres subcriterios.

\subsubsection{Criterio 2: Elección de la herramienta matemática}

De los cuarenta y dos reportes entregados, treinta y cuatro (81\%) incluyen una resolución utilizando la RTT (criterio 2.2), de los cuales veintiocho (82\%) integran el lenguaje del contexto en el procedimiento de resolución y en el uso de la fórmula trigonométrica tangente (subcriterio 2.2.1), cinco reportes (15\%) usan el lenguaje escolar y el 
lenguaje del contexto por separado (subcriterio 2.2.2), y en un reporte (3\%) sólo se menciona haber usado la razón trigonométrica, pero no se desarrolla el procedimiento de resolución.

Sólo dos profesores (5\%) reportan haber usado razones y proporciones (criterio 2.1) para resolver la situación, y ambos integran el lenguaje del contexto con el uso de sus herramientas matemáticas (subcriterio 2.1.1). En seis reportes (14\%) se incluyen solo datos y resultados, sin expresar o mostrar con qué herramienta matemática resolvieron la situaciónproblema (complemento a los treinta y seis reportes que cumplieron con el subcriterio 1.2).

\subsubsection{Criterio 3: Objetivo de la resolución}

El tercer criterio de análisis considera hacer una distinción entre la tendencia de los profesores a resolver la situación-problema, obteniendo sólo las alturas aproximadas (calculadas desde cada posición), o la tendencia a promediarlas para obtener la altura, además de calcular cada una. Ocho reportes (19\%) muestran sólo el cálculo de las tres alturas. En treinta y dos reportes (76\%) se presenta la promediación de las alturas, aunque en tres de ellos los profesores descartan una de las tres alturas porque se aleja o es muy diferente a las otras, por lo que promedian las dos más cercanas entre ellas.

Un reporte sólo describe las actividades realizadas en equipo y declara haber calculado las alturas, sin mencionar haberlas promediado; sin embargo, no reporta las medidas encontradas. Otro reporte, que igualmente describe todo sin reportar cálculos y resultados, señala que con la información recabada se encuentra la altura del objetivo, pero no dice explícitamente haber calculado el promedio de las alturas calculadas.

\subsubsection{Criterio 4: Naturaleza del modelo geométrico}

Este criterio se adentra en la naturaleza del saber en juego y pone de manifiesto el uso escolar de las nociones de razón y de proporción en la Trigonometría. A pesar de que el diseño solicita explícitamente un modelo a escala de lo observado, como instrucción intencional para permitir la transición de lo macro a lo micro, ningún reporte contiene triángulos o dibujos a escala. Once reportes (26\%) hacen uso de triángulos como representaciones ilustrativas de la situación problema y uno de ellos dibuja un sólo triángulo como referencia para las fórmulas trigonométricas que utilizará en el cálculo de los tres casos, por lo que sería el único en no ser considerado para el subcriterio 4.1.2 como el resto. 
En veinte reportes (48\%) encontramos dibujos de la situación, de los cuales dieciséis son ilustraciones (no representaciones a escala) a las que sobreponen los elementos de los triángulos como referencia para el cálculo de la altura en cada uno de los tres casos (a 3, 2 o 1 metro de distancia). Los cuatro dibujos restantes hacen uso de una ilustración genérica para todos los casos (Figura 6).

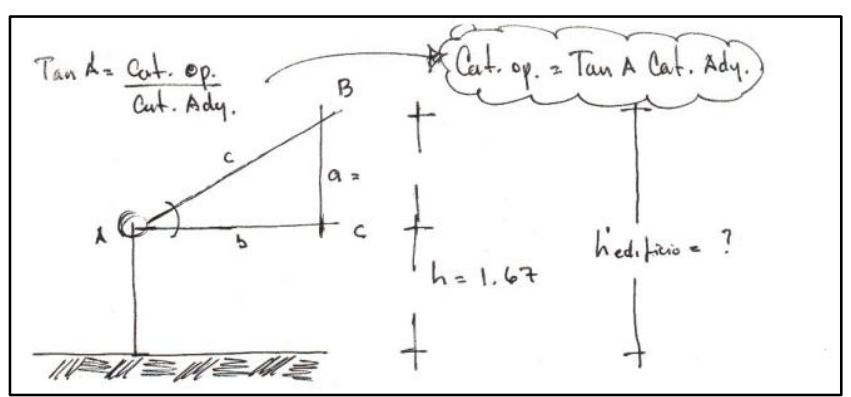

Figura 6- Ejemplos de ilustraciones genéricas como modelo geométrico de la situación

Ocho reportes (19\%) usan ambos tipos de representaciones ilustrativas, triángulos y dibujos de la situación, y se percibe que el modelo solicitado es el dibujo, mientras que los triángulos son apoyos visuales para el cálculo del valor faltante (Figura 7).

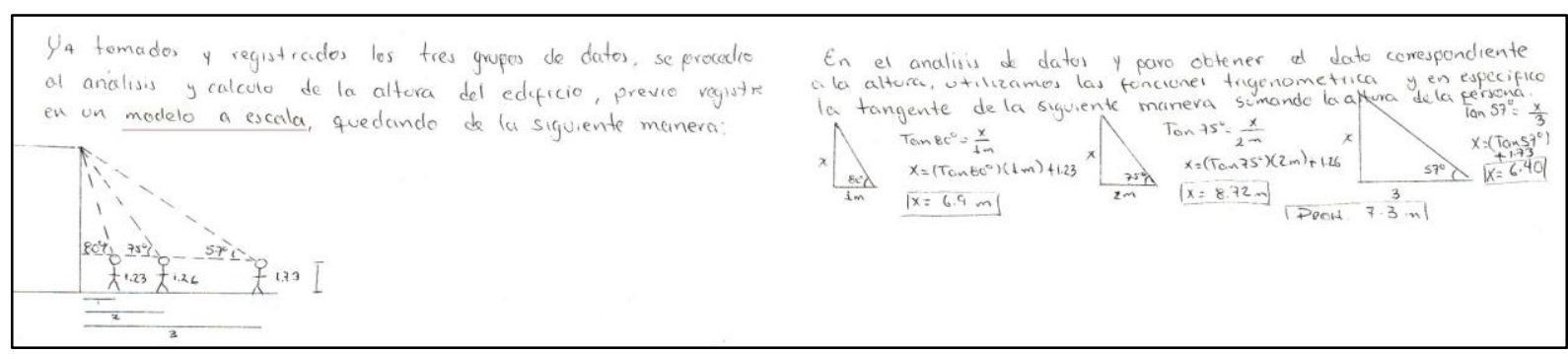

Figura 7- Ejemplo de un reporte que utiliza un dibujo como modelo geométrico y los triángulos como referentes para los cálculos de los valores faltantes (Reporte R21)

Finalmente, tres profesores (7\%) no hacen uso de triángulos o dibujos en sus reportes.

\subsubsection{Criterio 5: Relación Ángulo-Distancia}

El quinto criterio de análisis busca identificar qué es lo trigonométrico en las relaciones que establecen los profesores en el conjunto de todas las actividades que reflejan en sus reportes, tomando como punto de partida la distinción que plantea Wenthworth (1883, p. 3) entre la Geometría y la Trigonometría:

La geometría muestra en forma general que los lados y los ángulos de un triángulo son mutuamente dependientes. La Trigonometría comienza mostrando la naturaleza exacta de esta dependencia en el triángulo rectángulo y para este propósito emplea las razones de sus lados.

Encontramos nueve reportes $(21 \%)$ que no indican una relación lineal entre el ángulo y la distancia a la que se sitúan del objetivo (Figura 8), once reportes (26\%) que la establecen 
entre dos ángulos-distancias (Figura 9); y diez y siete reportes (40\%) que la establecen entre los 3 ángulos-distancia (Figura 10). Tres profesores no reportan las medidas del ángulo, aunque declaran haberlos medido; y en otros dos de los reportes no hicieron las mediciones porque utilizaron triángulos semejantes a partir de la inclinación del tubo dirigido al objetivo.

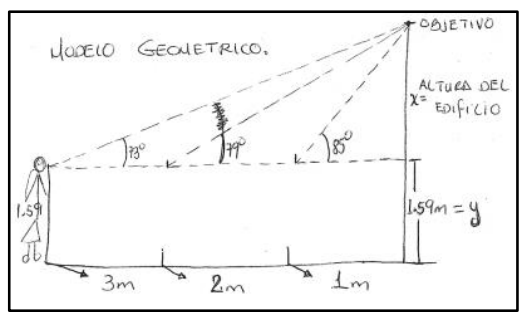

Figura $8-85^{\circ}$ a $1 \mathrm{~m}, 79^{\circ}$ a $2 \mathrm{~m}$ y $73^{\circ}$ a $3 \mathrm{~m}$

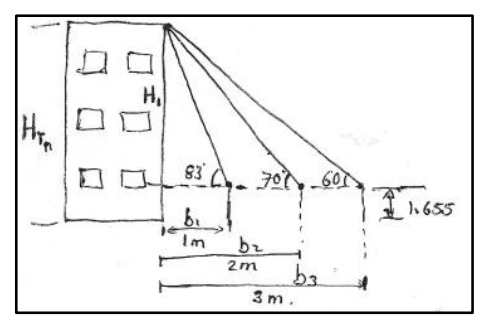

Figura 9- $83^{\circ}$ a $1 \mathrm{~m}, 70^{\circ}$ a $2 \mathrm{~m}$ y $60^{\circ}$ a $3 \mathrm{~m}$

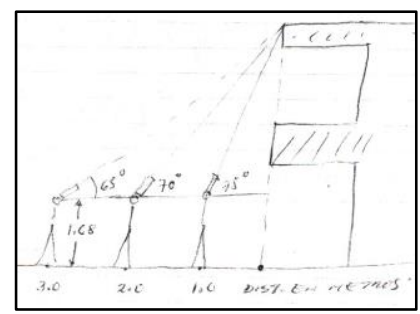

Figura $10-75^{\circ}$ a $1 \mathrm{~m}, 70^{\circ}$ a $2 \mathrm{~m}$ y $65^{\circ}$ a $3 \mathrm{~m}$

\section{Análisis y discusión}

\subsection{Análisis del discurso matemático escolar}

En el sistema escolar mexicano se introducen, por primera vez, las razones trigonométricas en el tercer año de la educación secundaria, cuando los alumnos tienen entre 14 y 15 años de edad. Los programas y planes de estudio de este nivel están regulados centralmente por la SEP y en la Reforma del 2006 a la Educación Secundaria (SEP, 2006, p. 132) se establece, en términos de conocimientos y habilidades, qué es lo que los alumnos de este nivel deben saber sobre la razón trigonométrica:

\footnotetext{
Reconocer y determinar las razones trigonométricas en familias de triángulos rectángulos semejantes, como cocientes entre las medidas de los lados. Calcular medidas de lados y de ángulos de triángulos rectángulos a partir de los valores de razones trigonométricas. Resolver problemas sencillos, en diversos ámbitos, utilizando las razones trigonométricas.
}

Este momento, en el que un estudiante tiene su primer contacto con la Trigonometría, las razones trigonométricas son trabajadas como cocientes entre medidas que permiten calcular un valor faltante en el triángulo rectángulo. En particular, las orientaciones didácticas, ponen atención en utilizar estos cocientes para calcular la medida del ángulo y dado que se contextualiza en la resolución del triángulo su medida se da naturalmente en grados.

En la revisión de la organización didáctica, entendida ésta como la secuenciación de actividades, conceptos y ejercicios; de algunos de los libros de texto aprobados por la SEP (FILLOY et al., 2009; GARCÍA; MENDOZA, 2008; BRISEÑO et al., 2008; MANCERA, 2008) se reconocieron las siguientes regularidades: 
i. Se inicia con la medición de ángulos y longitudes de los lados de distintos triángulos, para determinar si se trata de triángulos rectángulos, y entonces reconocer regularidades entre ellos (por ejemplo: Figura 13).

ii. Se identifican la congruencia entre ángulos y la semejanza entre triángulos, para establecer las razones proporcionales entre las medidas de los lados, en relación a un ángulo (por ejemplo: Figura 14).

iii. Se establecen las razones trigonométricas a partir de las regularidades encontradas en los cocientes anteriores.

iv. Se resuelven triángulos rectángulos (cálculo de un valor faltante, ya sea longitud de un lado o medida de un ángulo).

v. Se resuelven problemas de aplicación, como el cálculo de áreas de polígonos regulares y el cálculo de distancias inaccesibles, éstas últimas presentadas como situaciones hipotéticas a resolver en papel.

Las actividades enmarcadas en las dos primeras regularidades están situadas en un contexto de proporcionalidad, y son las que direccionan el resto de las actividades en el tema y donde comienzan a construirse los significados asociados a lo trigonométrico en la escuela. Sin embargo, lo proporcional está jugando un rol de condición inicial en la que se establecen las reglas a seguir para usar las razones trigonométricas; por lo que el profesor podría estar obviando lo proporcional si la tarea le demanda el uso de dicha razón.

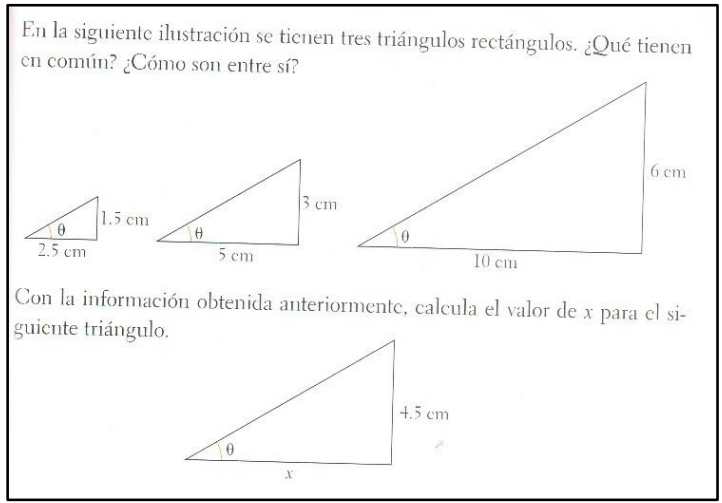

Figura 11- Fuente: Briseño et al. (2008)

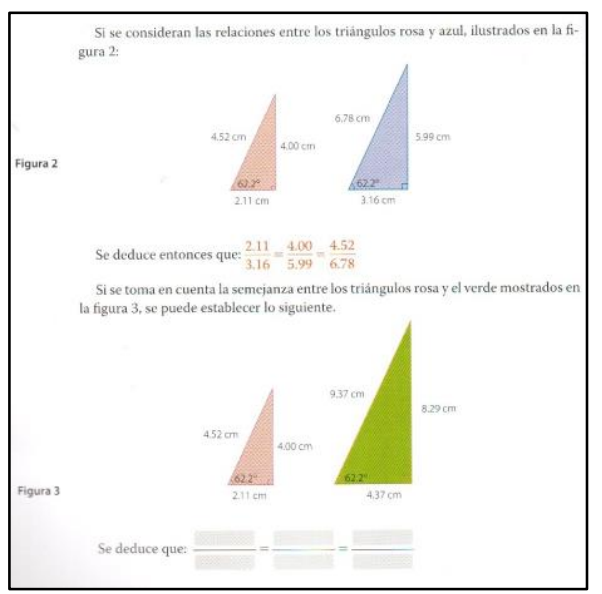

Figura 12- Fuente: Mancera (2008)

Las regularidades, en el triángulo, de donde surgen las razones trigonométricas se establecen en relación a un mismo ángulo (ver por ejemplo, Figuras 11, 12 y 13), con base en lo cual adquiere sentido hablar de razón cuando se establecen las razones trigonométricas. Para identificar la razón constante en relación a un ángulo en particular se utiliza un lenguaje geométrico-proporcional (triángulos rectángulos, triángulos semejantes, razones y 
proporciones), sin embargo, los triángulos no se construyen sino que se le proporcionan al estudiante para que sean medidos, en el mejor de los casos, o se identifiquen las medidas correspondientes al ángulo, los catetos y la hipotenusa. Algunos textos pasan de la expresión proporcional al cálculo de cocientes para establecer las razones trigonométricas (Figura 13).

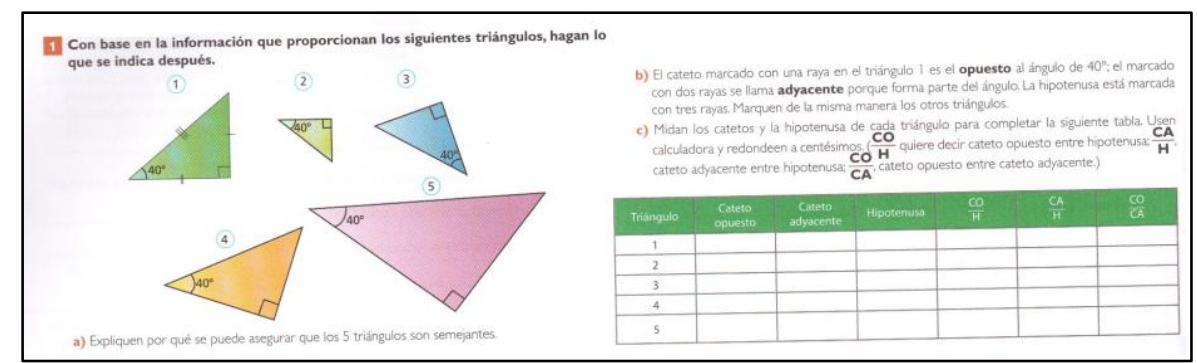

Figura 13- Fuente: García y Mendoza (2008)

Resulta, entonces, que la norma, identificada por Mesa y Herbst (2011), en la que el instructor proporciona al estudiante toda la información necesaria para resolver los problemas y obtener resultados con base en la repetición de un procedimiento, se puede identificar también en los libros de texto. Ejemplo claro de esta norma se presenta en los problemas de aplicación.

Las Figuras 14 y 15 son ilustraciones de problemas de aplicación, en las que se sobreponen los trazos y las medidas necesarias para convertirlo en un problema de resolución de triángulo, igual a los resueltos previamente en el tema. No es necesario que el estudiante haga el trazo, calcule medidas y obtenga una solución por otra vía; y en ese sentido el estudiante no tiene el control de lo que hace, no justifica, ni comprueba que su procedimiento sea el apropiado para dar respuesta al problema; con lo que se manifestarían las otras dos normas identificadas por Mesa y Herbst (2011). Llama la atención que algunos textos incluyen en la introducción o en la conclusión del tema alguna reflexión sobre las aplicaciones y la importancia de la Trigonometría, y ponen atención en la medición de realidades no manipublables (Figura 16 ).

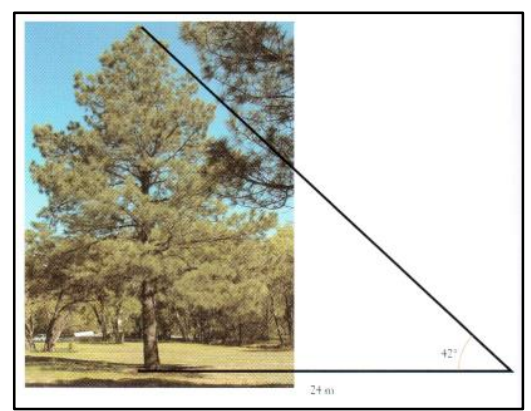

Figura 14- Fuente: Briseño et al. (2008)

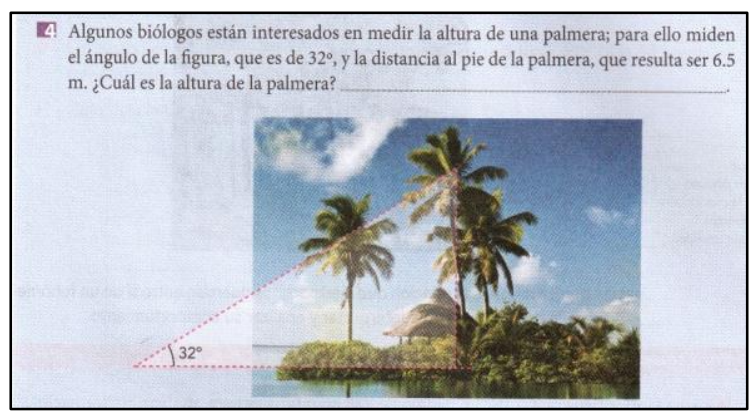

Figura 15- Fuente: Filloy et al. (2009) 
Tanto en este tipo de ilustraciones como en las figuras geométricas los libros utilizan, frecuentemente, medidas que no son reales; lo que las convierte sólo en información necesaria para sustituir en la fórmula que resuelve el problema.

Finalmente, encontramos en uno de los textos un cuadro sintético de las fórmulas para calcular valores trigonométricos que, aun estando en el tema de Razones Trigonométricas y de usar sus expresiones en forma de razones, denominan como Funciones Trigonométricas (Figura 16) sin hacer reflexión sobre la naturaleza de cada una (razón o función).

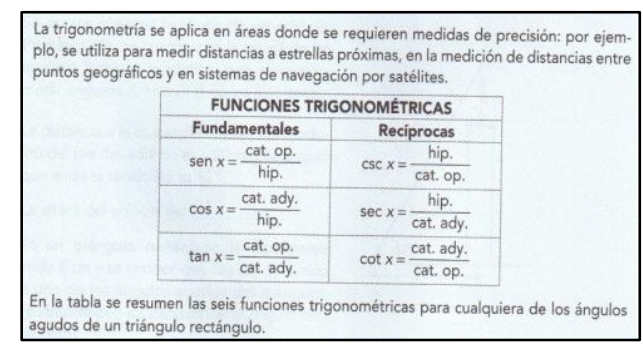

Figura 16- Fuente: Filloy et al. (2009)

A simple vista podría considerarse un detalle mínimo en la nomenclatura, pero considerando las dificultades reportadas en la literatura (GRABOVSKIJ; KOTEL'NIKOV 1971; DE KEE; MURA; DIONNE, 1996; KENDAL; STACEY, 1998) las implicaciones no son triviales; sobre todo tomando en cuenta que posteriormente, al trabajar con las funciones trigonométricas éstas se expresarán, por ejemplo, como $f(x)=\operatorname{sen} x$, estarán definidas en los reales y se despojarán de los elementos geométricos que son fundamentales para trabajar con las razones trigonométricas.

El análisis del programa y los libros de texto nos da una primera pauta para reconocer un valor epistémico pragmático atribuido al campo de la Trigonometría en el dME, que se extiende incluso a niveles educativos superiores. Por ejemplo, se considera fundamental para muchas aplicaciones de arquitectura, astronomía y física, complemento al estudio de la geometría analítica y un antecedente indispensable para el cálculo diferencial e integral (DE OTEYZA et al., 2008); o uno de los primeros tópicos matemáticos que vincula el razonamiento algebraico, el geométrico y el gráfico, y precursor importante para el entendimiento del pre-cálculo y el cálculo (WEBER, 2005). La Trigonometría es vista en estrecha relación con otras áreas, matemáticas y científicas; pero esencialmente al servicio de otros conocimientos o, como señalan Mesa y Herbst (2011), como proveedor de habilidades y conocimiento técnico.

Con base en lo anterior, planteamos que la racionalidad práctica del profesor si bien mantiene las normas del contrato, emerge de las bases de comunicación de la Trigonometría 
escolar (incluida la que él estudió) o, dicho en términos de nuestro fundamento teórico, del discurso trigonométrico escolar (dTE). Este discurso estaría normando toda la actividad didáctica referida a lo trigonométrico, incluidas las reformas educativas y la innovación didáctica.

\subsection{Análisis de las producciones del profesor, a la luz del dTE}

Es posible explicar que los profesores signifiquen lo trigonométrico como una relación lineal entre el ángulo y las distancias desde donde se posicionan frente al edificio, a partir de la ilusión de la linealidad, ampliamente documentada por De Bock et al. (2007). Sin embargo, al problematizar el saber matemático en juego es necesario entender el papel que juega la Trigonometría en el fenómeno identificado, lo que no descarta el interés por entender por qué la linealidad se extrapola a lo trigonométrico. De hecho, es a partir de nuestro análisis del dME que reconocemos que en la Trigonometría escolar no se presentan explicaciones y actividades que permitan reconocer e identificar que lo trigonométrico no está en la relación ángulo-distancia, sino en la naturaleza de dicha relación, y éste constituye un espacio de oportunidad para que la linealidad se manifieste.

Comenzaremos el análisis de las producciones del profesor identificando la ruptura del contrato didáctico que provoca la situación-problema al no proporcionarle toda la información para resolverla en papel, en ese sentido juega un doble papel de profesor-estudiante, pues atiende a las indicaciones del instructor para llevar a cabo una actividad no convencional y plasma en su reporte el dominio que tiene de los saberes en juego.

\subsubsection{Organización y objetivo didácticos}

Se asumió que el denominar a la tangente de distintas maneras (como razón, función, procedimiento, fórmula etc.) era común en el profesor de nivel medio superior por la regularidad con la que él trabaja las funciones, aun cuando sólo esté utilizando una razón en la resolución de la situación-problema. Sin embargo, esta misma indistinción se encontró en uno de los libros de textos, y Montiel y Buendía (2013) lo identificaron también en un programa de estudios; lo que señala una falta de claridad entre la naturaleza de una u otra noción matemática, provocada por el énfasis puesto en el procedimiento matemático más que en el concepto. Este énfasis se identifica como la vía para adquirir habilidad con las técnicas, la 
cual se manifiesta cuando el estudiante logra resolver tareas similares a las resueltas por el profesor en clase. Ejemplo de esta situación en los textos se muestra en la Figura 12.

Mesa y Herbst (2011) reconocen que es deber y prerrogativa del instructor decir que los estudiantes han aprendido un cierto objeto de conocimiento, pero, para hacerlo, el profesor necesita confiar en cierta evidencia de parte de los estudiantes (por ejemplo, completar una tarea). La organización didáctica de los libros de texto plantea como invariante la tarea de encontrar un valor faltante en el triángulo rectángulo, proporcionando toda la información necesaria para lograrlo. En la experiencia que estamos analizando, si bien la situaciónproblema no proporciona toda la información, el profesor la obtiene y da evidencia del cumplimiento de la tarea obteniendo un valor, es decir, la altura; lo cual explica la promediación de las tres alturas aunque explícitamente no se le solicitara.

Identificamos otra ruptura del contrato, en la resolución de la situación-problema, al trabajar con el modelo geométrico. Mientras que las definiciones y las actividades en los textos analizan las regularidades en las relaciones de las partes de un triángulo con relación a un mismo ángulo, los modelos geométricos que hacen los profesores no constituían triángulos semejantes, cada razón trigonométrica se establecía en relación a un ángulo en particular. Es decir, mientras que en la costumbre escolar se relacionan siempre triángulos con triángulos (semejantes) en el papel, la situación-problema requería relacionar la realidad con el triángulo construido. De ahí que en los textos los triángulos de distintos tamaños (como en las Figuras 11, 12 y 13) puedan representar una misma realidad porque son semejantes, no así los triángulos como los que se presentan en el reporte R16 (Figuras 17, 18 y 19), en donde se puede identificar una influencia de las distancias a las que debe situarse el profesor del objetivo a medir (a $3 \mathrm{~m}, 2 \mathrm{~m}$ y $1 \mathrm{~m}$ ) para trazar la base de los triángulos. Sin embargo, al realizarlos el profesor no cuida el que, sin importar esta posición, la altura del objetivo y por lo tanto la de los triángulos, debe ser la misma.

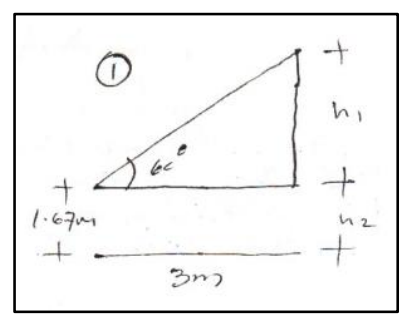

Figura 17- Modelo del cálculo de alturas a $3 \mathrm{~m}$ del objetivo

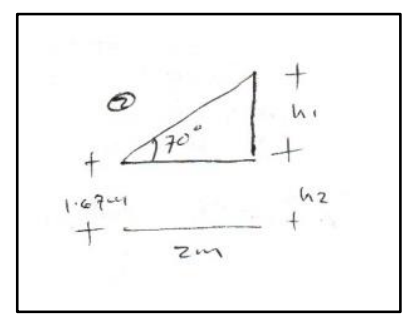

Figura 18- Modelo del cálculo de alturas a $2 \mathrm{~m}$ del objetivo

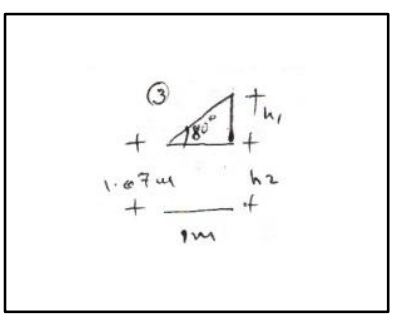

Figura 19- Modelo del cálculo de alturas a $1 \mathrm{~m}$ del objetivo

\subsubsection{Construcción del modelo geométrico}


El uso de ilustraciones representativas o referencias se presenta tanto en las producciones del profesor como en los libros de texto, costumbre escolar que no resulta problemática en la resolución de lo que el profesor y los libros consideran tareas trigonométricas, de hecho puede prescindirse de ellas porque no son una construcción geométrica mediadora entre el problema y la solución.

Los textos revisados incluyen, en los ejercicios de aplicación, problemas de cálculo de distancias inaccesibles en los que se proporcionan las medidas del ángulo de depresión o elevación, según sea el caso, y de la distancia que sí se conoce. Es común que en el dibujo que representa la situación a medir se sobrepongan los triángulos con las distancias conocidas, y esto permite elegir, con base en la memorización, la relación trigonométrica a utilizar (Figuras 14 y 15). Es decir, no hay en sí un análisis de la situación, sino del triángulo y éstos no son construcciones geométricas en el sentido estricto. Esto explica por qué no hay modelos a escala, aunque así los llame el profesor (Figura 7). Una característica invariante del dTE es su abandono de las construcciones geométricas propias de las actividades que le anteceden en el Plan de Estudios.

\subsubsection{Significado lineal en la relación ángulo-distancia}

Es cierto que las medidas angulares son aproximaciones poco precisas por que fueron hechas con un pequeño tubo de cartón y un transportador, pero también es cierto que de haber construido modelos a escala, como lo pedía la situación-problema, habría sido evidente la imposibilidad de hacerlos con esas medidas. Esta habría sido, quizá, la ruptura del contrato que más detonara una discusión respecto de la concepción que tienen los profesores sobre lo trigonométrico en el triángulo rectángulo.

En este sentido, postulamos, un pensamiento trigonométrico referido a las razones en el triángulo rectángulo reconocería que no es posible la construcción de triángulos como los de las Figuras 1, 4, 9 y 10. De poder establecer esa relación entre ángulo-cateto no serían necesarias las razones trigonométricas.

Sin embargo, sin más casos en los que el profesor manifieste esta relación u otros datos provenientes de entrevistas o actividades de otro tipo tenemos que considerar, también, que establecer esta relación se debe a que en el dTE no se estudia, no es relevante. Por este motivo la ilusión de la linealidad es insuficiente para explicar la manifestación de este significado en los reportes de los profesores. 


\section{Reflexiones finales}

En la experiencia con los profesores el triángulo, como modelo geométrico, no es una construcción geométrica, en tanto no guarda una relación proporcional con la realidad modelada. De haber sido así, el profesor encontraría imposible utilizar las medidas de $60^{\circ}$, $50^{\circ}$ y $40^{\circ}$ para los ángulos indicados. Esta relación entre el ángulo y la distancia no es lineal, no es cuadrática, no es logarítmica, ni exponencial. Es aquí donde reconocemos lo trigonométrico de la construcción del modelo y la necesidad de utilizar la semejanza entre triángulos para estudiar la naturaleza de estas relaciones particulares. Sin embargo, para el discurso trigonométrico escolar la semejanza resulta ser una condición inicial sobre la que se sitúa el tema y en consecuencia todo lo que sigue a ella la cumple, no hay necesidad de corroborarla. Es decir, la construcción geométrica es innecesaria y se concentra la actividad matemática en la operación aritmética para la obtención del valor faltante, lo que aunado a la falta de atención y/o reconocimiento de lo que es trigonométrico en la relación ángulo-lado del triángulo es lo que identificamos como el fenómeno de la aritmetización trigonométrica. Este énfasis en lo numérico, para el tratamiento de la razón y la proporción, fue reportada por Oller y Gairín (2013); y su perspectiva de análisis puede abonar a nuestra investigación en tanto el concepto matemático de nuestro interés es tratado en la escuela como razón proporcional. En este momento, con base en la fundamentación teórica construida en el presente documento y la evidencia empírica mostrada, reconocemos que este fenómeno es un efecto de la pérdida del proceso geométrico en la construcción de lo trigonométrico, donde las razones trigonométricas se convierten en el proceso aritmético de dividir las longitudes de los lados del triángulo, lo cual, además, se manifiesta de manera clara en la estructuración del discurso escolar.

La problematización de la Trigonometría escolar, desde el enfoque teórico de la Socioepistemología, nos permitió ver más allá de la propuesta del plan de estudios o de la organización didáctica de los libros de texto, para identificar lo que subyace y denominamos discurso trigonométrico escolar, y que ha permanecido invariante aun en el transcurso de 
varias reformas educativas: lo trigonométrico está en las razones trigonométricas, en la técnica para calcular un valor.

Partimos de asumir que la razón trigonométrica, como herramienta, si bien resuelve el problema de calcular la altura del edificio (cumpliendo así con el objetivo escolar de elegir correctamente la razón trigonométrica tangente y calcular el valor faltante), no asegura un pensamiento trigonométrico ante el manejo del triángulo, sus elementos y las relaciones entre estos. Por ello no centramos nuestra atención en el manejo del objeto matemático (la razón trigonométrica), sino en la práctica que demanda modelar una realidad macro no manipulable, cuantificando la inclinación haciendo uso de ángulos, midiendo distancias y trazando proyecciones al construir triángulos, así como de la construcción de modelos geométricos haciendo uso de la semejanza y las herramientas que se requieran para representar la situación vivida con el objetivo de estudiarla.

Con base en lo anterior, no podemos declarar que el profesor no domina los conceptos o tiene concepciones erróneas, sino que hay significados de lo trigonométrico que subyacen a su quehacer: significado lineal, significado como división de longitudes, significado como técnica para obtener un valor; porque subyacen también a la Trigonometría escolar y en consecuencia a todo aquello que la transmite con intencionalidad didáctica.

\section{Referencias}

BRISEÑO, L.; CARRASCO, G.; MARTÍNEZ, P.; PALMAS, O.; STRUCK, F.; VERDUGO, J. Matemáticas 3. México, DF: Santillana Integral, 2008.

BUENDÍA, G.; MONTIEL, G. From history to research in mathematics education: Socioepistemological elements for trigonometric functions. En: KATZ, V.; TZANAKIS, C. (Ed.). Recent developments on introducing a historical dimension in Mathematics Education. Washington, DC, USA: Mathematical Association of America, 2011, p. 67-82.

CANTORAL, R.; FARFÁN, R. M.; LEZAMA, J.; MARTÍNEZ, G. Socioepistemología y representación: algunos ejemplos. Revista Latinoamericana de Investigación en Matemática Educativa. México, DF, v. 9, n. 4, p. 83-102, Octubre. 2006.

CASTAÑEDA, A.; ROSAS, A.; MOLINA, G. El discurso matemático escolar de los logaritmos en los libros de texto. Premisa. Buenos Aires, Argentina, v. 12, n. 44, p. 3-18, Febrero. 2010.

DE BOCK, D.; VAN DOOREN, W.; JANSSENS, D.; VERSCHAFFEL, L. The Illusion of Linearity: from analysis to improvement. New York, USA: Springer. 2007.

DE KEE, S.; MURA, R. ; DIONNE, J. La comprensión des notions de sinus et de cosinus chez des élèves du secondaire. For the Learning of Mathematics, Fredericton, NB, Canada, v.16, n. 2, p. 19 27, Julio. 1996. 
DE OTEYZA, E. (Coord.); LAM, E.; HERNÁNDEZ-GARCIADIEGO, C.; CARRILLO, A.; RAMÍREZ, A. Geometría Analítica y Trigonometría, México, DF: Pearson Educación. 2008.

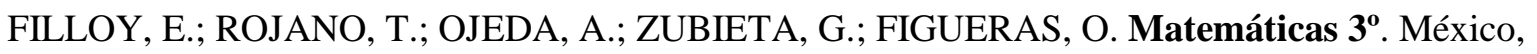
DF: Ediciones Pedagógicas / McGraw-Hill. 2009.

GARCÍA, S.; MENDOZA, T. Fractal 3. México, DF: Ediciones SM. 2008.

GRABOVSKIJ, M.; KOTEL'NIKOV, P. The use of kinematic models in the study of trigonometric functions. Educational Studies in Mathematics, Nertherlands, v. 3, n. 2, p. 147-160, Abril. 1971.

HERBST, P.; CHAZAN, D. Research on practical rationality: studying the justification of actions in mathematics teaching. The Mathematics Enthusiast, Charlotte, NC, v. 8, n. 3, p. 405-462, Julio. 2011.

JÁCOME, G. Estudio socioepistemológico de la razón trigonométrica. Elementos para la construcción de su naturaleza proporcional. 2011. 170 f. Tesis (Maestría en Ciencias en Matemática Educativa) - Centro de Investigación en Ciencia Aplicada y Tecnología Avanzada, Unidad Legaria, del Instituto Politécnico Nacional, México, DF, 2011.

KENDAL, M.; STACEY, K. Teaching trigonometry. Two methods of introducing trigonometry. Australian Mathematics Teacher, Adelaide SA, v. 54, n. 1, p. 34-39, Abril. 1998.

KILPATRICK, J.; HOYLES, C.; SKOVSMOSE, O.; VALERO, P. Meanings of meaning of mathematics. En: KILPATRICK, J.; HOYLES, C.; SKOVSMOSE, O.; VALERO, P. (Ed.). Meaning in Mathematics Education. New York, USA: Springer, 2005. p. 9-16.

MALDONADO, E. Un análisis didáctico de la función trigonométrica. 2005.73 f. Tesis (Maestría en Ciencias con Especialidad en Matemática Educativa) - Centro de Investigación y de Estudios Avanzados del Instituto Politécnico Nacional, D.F., México, 2005.

MANCERA, E. Matemáticas 3. México, DF: Santillana Ateneo. 2008.

MESA, V.; HERBST, P. Designing representations of trigonometry instruction to study the rationality of community college teaching. ZDM The International Journal on Mathematics Education, Berlin, v. 43, n. 1, p. 41-52, Febrero. 2011.

MONTIEL, G. Construcción de conocimiento trigonométrico. Un estudio socioepistemológico. México, DF: Ediciones Díaz de Santos. 2011.

MONTIEL, G.; BUENDÍA, G. Desarrollo del Pensamiento Funcional-Trigonométrico. En: FERRARI, M.; MARTÍNEZ, G.; BUENDÍA, G. (Coords.). Resignificación de funciones para profesores de matemáticas. México, DF: Ediciones Díaz de Santos, 2013, 169-205.

OLLER, A.; GAIRÍN, J. La génesis histórica de los conceptos de razón y proporción y su posterior aritmetización. Revista Latinoamericana de Investigación en Matemática Educativa, México, DF, v. 16, n. 3, p. 317-338, Noviembre. 2013.

PEDERSEN, O. Logistics and the theory of functions. An essay in the history of Greek mathematics. Archives Internationales D'Histoire des Sciencies, Turnhout, v. 24, n. 94, p. 29-50, Junio. 1974.

SECRETARÍA DE EDUCACIÓN PÚBLICA. Educación básica. Secundaria. Programas de estudio 2006. México, DF: Comisión Nacional de Libros de Texto Gratuitos. 2006. 
SOTO, D. El Discurso Matemático Escolar y la Exclusión. Una Visión Socioepistemológica. 2010. 105 f. Tesis (Maestría en Ciencias con Especialidad en Matemática Educativa) - Centro de Investigación y de Estudios Avanzados del Instituto Politécnico Nacional, D.F., México, 2010.

VAN BRUMMELEN, G. The mathematics of the heavens and the earth. The early history of trigonometry. New Jersey, USA: Princeton University Press. 2009.

WEBER, K. Teaching trigonometric functions: Lessons learned from research. Mathematics Teacher, Reston, Virginia, v. 102, n. 2, p. 144-150, Septiembre. 2008.

WEBER, K. Student's understanding of trigonometric functions. Mathematics Education Research Journal, Australia, v. 17, n. 3, p. 91-112, Octubre. 2005.

WENTHWORTH, G. A. Plane and Spherical Trigonometry. Boston, USA: Ginn, Heath \& Co. 1883.

Submetido em Junho de 2013. Aprovado em Dezembro de 2013. 